\title{
Monoamine Oxidase Inhibitors: Nature of Their Interaction with Rabbit Pancreatic Islets to Alter Insulin Secretion*
}

\author{
J.M. Feldman and B. Chapman \\ Durham Veterans Administration Hospital and Division of Endocrinology, Dept. of Medicine, Duke Univ. Medical Center, Durham, \\ North Carolina, U.S. A.
}

Received: May 5, 1975, and in revised form: July 15, 1975

\begin{abstract}
Summary. Using an in vitro rabbit pancreas system, we studied the effect of monoamine oxidase (MAO) inhibitors on glucosestimulated insulin secretion. We evaluated the effect of both brief $(15 \mathrm{~min})$ and prolonged $(60 \mathrm{~min})$ exposure of pancreas segments to non-hydrazine (harmine, $\alpha$-methyltryptamine, tranylcypromine and pargyline) and hydrazine (phenelzine, nialamide, iproniazid) type MAO inhibitors. All of the hydrazine type MAO inhibitors potentiated glucose-stimulated insulin secretion. Of the non-hydrazine inhibitors, only harmine and $\alpha$-methyltryptamine potentiated glucose-stimulated insulin secretion. Hydrazine, although not itself an MAO inhibitor, also potentiated insulin secretion. Sixty minutes of exposure to tranylcypromine or $\alpha$-methyltryptamine caused a decrease in insulin secretion. These MAO inhibitors are primary amines and primary amines can inhibit insulin secretion. The dopamine (DA) or serotonin (5-HT) content of the B-cells was increased by incubating rabbit pancreas with L-3, 4-dihydroxyphenylalanine (L-Dopa) or 5-hydroxytryptophan (5-HTP) for forty-five minutes
\end{abstract}

prior to stimulation with glucose. Non-hydrazine MAO inhibitors increased dopamine inhibition of insulin secretion and either did not alter, or decreased serotonin inhibition of insulin secretion. Rabbit pancreatic islets were isolated using the collagenase digestion technique. The MAO activity of islet homogenates was determined using 5-HT and DA as substrates. Rabbit islet MAO has only one-tenth the specific activity against 5 -HT $(35 \pm 8.7 \mu \mu \mathrm{moles} / \mathrm{mg} /$ $\min , \mathrm{M} \pm \mathrm{SEM})$ that it has against DA (357 $\pm 62.3 \mu$ umoles $/ \mathrm{mg} /$ $\mathrm{min}$ ). This suggests that one reason that MAO inhibitors do not increase serotonin inhibition of insulin secretion is because MAO is not the major pathway for $5-\mathrm{HT}$ inactivation in rabbit pancreatic islets. These studies suggest that MAO inhibitors alter insulin secretion, by both decreasing B-cell monoamine degradation and by mechanisms that do not involve MAO inhibition.

Key words: Insulin secretion, monoamine oxidase, monoamine oxidase inhibitors, islets of Langerhans, serotonin, dopamine.

\section{Introduction}

Dopamine and serotonin are potent inhibitors of insulin secretion [1]. The presence of these monoamines in the Islets of Langerhans of the pancreas raises the possibility that they may play a role in the regulation of insulin secretion [2]. If dopamine and serotonin play such a role, it is reasonable to expect a mechanism to be present in the islets for the inactivation of these monoamines. Monoamine oxidase (monoamine: oxygen oxidoreductase, 1.4.3.4), an enzyme that inactivates dopamine and serotonin in other tissues, is present in the islets of many species $[3,4]$.

Chemicals that inhibit monoamine oxidase (MAO inhibitors) have been reported to both increase [5-7] and to decrease [8-11] insulin secretion. These variable effects may be due to the different species studied (rats, mice and rabbits), the different experimental designs used (in vivo and in vitro studies) and the different types of MAO inhibitors used (reversible and irreversible).

\footnotetext{
* Supported by the Veterans Administration (2650-1).
}

In the present study we have evaluated the effect of varying concentrations of reversible and irreversible MAO inhibitors on insulin secretion. We have used MAO inhibitors that are hydrazine derivatives and MAO inhibitors that are not hydrazine derivatives. Finally, we have studied the interaction of a variety of MAO inhibitors with intracellular dopamine and intracellular serotonin. Our studies suggest that MAO inhibitors alter insulin secretion both by inhibiting MAO, and by other pharmacological actions that do not involve MAO inhibition.

\section{Materials and Methods}

\section{Pancreas Incubation System}

A previously described in vitro rabbit pancreas system was used in these studies [12]. Four hours after the food was removed from their cages, male New Zealand white rabbits weighing 1 to $2 \mathrm{~kg}$ were anesthetized with pentobarbital. The pancreas was removed and cut into segments weighing 15 to $25 \mathrm{mg}$. Two types of incubation studies were performed. In the first type, the segments are preincubated in $2 \mathrm{ml}$ of 
modified Krebs Ringer Bicarbonate (KRB) buffer, with a nonstimulatory concentration of glucose (3.3 $\mathrm{mM}$ ), sodium pyruvate $5 \mathrm{mM}$, sodium fumarate 5 $\mathrm{mM}$, sodium glutamate $5 \mathrm{mM}$ and bovine serum albumin $4 \mathrm{mg} / \mathrm{ml}$. The flasks were gassed with $95 \%$ oxygen- $5 \%$ carbon dioxide; the $\mathrm{pH}$ of the buffer was 7.40. After a thirty minute preincubation period the medium was discarded and the pieces transferred to flasks containing $2 \mathrm{ml}$ of fresh medium for an additional fifteen minute period (called basal observation). The pieces were then transferred to a third flask containing medium with glucose $16.5 \mathrm{mM}$ and the appropriate test substance(s) for another fifteen minute period (called stimulated observation).

In the second type of incubation study, the segments were either preincubated in modified KRB buffer alone or modified KRB buffer with the test substances under study for thirty minutes. The media was then discarded and the pieces were preincubated for an additional fifteen minutes in flasks containing $2 \mathrm{ml}$ of fresh medium of identical composition to that used in the first preincubation (called basal observation). The pieces were then incubated in a third set of flasks containing $2 \mathrm{ml}$ of fresh medium for another fifteen minutes (called stimulated observation). In addition to the test substances present during the first two incubations, the flasks also contained $16.5 \mathrm{mM}$ glucose. In these incubation studies the pancreas segments were exposed to the test substance(s) during the fortyfive minutes prior to glucose stimulation and during the fifteen minutes of glucose stimulation. This contrasts with the incubation procedure described in the previous paragraph in which the pancreas segments were exposed to the test substances only during the fifteen minute period in which they were stimulated with glucose.

All incubations were performed at $37^{\circ} \mathrm{C}$ in stoppered $10 \mathrm{ml}$ flasks in a gyrorotary shaker. At the conclusion of the basal and the stimulated incubation, aliquots of the incubation media were placed in chilled tubes and directly assayed for insulin by a double antibody radioimmunoassay technique [13]. Insulin release was measured in the basal incubation to ascertain that the study groups did not differ significantly from one another in their insulin secretion pattern, before stimulation with $16.5 \mathrm{mM}$ glucose. In the concentrations employed, the compounds used in the present study did not interfere with the radioimmunoassay measurement of insulin.

To compare the effect of different MAO inhibitors in the various studies, the insulin secretion of each pancreas segment incubated with an MAO inhibitor and/or L-dopa or 5-HTP was expressed as a percent of the mean insulin secretion of the control segments in the experiment. The mean insulin secretion of the control segments was obtained by incubating 7 pancreas segments in $16.5 \mathrm{mM}$ glucose alone during the final 15 min incubation period.

Enzyme Assay of Isolated Pancreatic Islets. Pancreatic tissue was obtained from male albino rabbits weighing 1 to $2 \mathrm{~kg}$. Pancreatic islets were isolated by collagenase digestion, following the protocol of Lacy and Kostianovsky [14]. One hundred to 150 islets were pooled, washed with Hanks' solution containing $3.3 \mathrm{mM}$ glucose [15], and homogenized. An aliquot of the homogenate was digested with $0.5 \mathrm{~N} \mathrm{NaOH}$ for 30 min at room temperature; the protein content of the homogenate was then determined by the Lowry method [16]. Aliquots of the tissue homogenates were also assayed for MAO activity using the radioassay method of McCaman et al. [17]. In this method tissue homogenates are incubated with ${ }^{14} \mathrm{C}$-labelled serotonin or dopamine. The aldehyde and acid metabolites of the monoamines are then isolated by differential solvent extraction. The MAO activity of the homogenates is equal to the amount of metabolites that are formed. Each measurement was corrected for the blank value by subtracting from it the results of an assay carried out by substituting an aliquot of previously boiled liver homogenate $\left(100^{\circ} \mathrm{C}\right.$ for $\left.20 \mathrm{~min}\right)$ for an aliquot of homogenate. The specific activity of the MAO was expressed as $\mu \mu$ moles product formed/ $\mathrm{mg}$ tissue protein/min incubation.

\section{Chemicals}

The following chemicals were purchased from commercial sources: L-3,4-dihydroxyphenylalanine (L-dopa), harmine $\mathrm{HCl}$, iproniazid phosphate, hydrazine dihydrochloride (Sigma Chemical, St. Louis Mo.), L-5-hydroxytryptophan (Calbiochem, La Jolla, $\mathrm{Cal}$ ), 3,4-dihydroxyphenylethylamine $\mathrm{HBr}$ (dopamine) (side chain- $1-{ }^{14} \mathrm{C}$ ) with specific activity $9.3 \mathrm{mCi} /$ mmole (New England Nuclear, Boston Mass.) serotonin creatinine sulfate (side chain-2- ${ }^{14} \mathrm{C}$ ) with specific activity of $55 \mathrm{mCi} / \mathrm{mmole}$ (Amersham Searle, Arlington Heights, Ill.).

The following chemicals were gifts: pargyline $\mathrm{HCl}$ (Abbott laboratories North Chicago, Ill.); tranylcypromine sulfate (Smith, Kline and French laboratory, Philadelphia, Pa.); alpha-methyltryptamine (Upjohn Co. Kalamazoo Mich.); phenelzine sulfate (WarnerLambert, Morris Plains, N.J.), nialamide (Pfizer Inc. Brooklyn, N.Y.).

\section{Statistical Methoas}

In each experiment the significance of the difference between means of the groups was determined by 
the Student's t-test or the paired t-test [18]. In some experiments analysis of variance and the Dunnett's test was also used [18].

\section{Rational of Study}

The purpose of the present study was to determine if MAO inhibitors alter pancreatic insulin secretion in the rabbit. Three different types of MAO inhibitors were evaluated in the present study [19]: harmine (HARM) and $\alpha$-methyltryptamine $(\alpha$-MT) are nonhydrazine reversible MAO inhibitors; tranylcypromine (TRAN) and pargyline (PARG) are non-hydrazine, irreversible MAO inhibitors; phenelzine (PHEN), nialamide (NIAL) and iproniazid (IPRO), all hydrazine derivatives, are irreversible MAO inhibitors. The reversible $\mathrm{MAO}$ inhibitors are maximally effective when they first contact the MAO. With the exception of TRAN, the irreversible inhibitors are maximally effective as MAO inhibitors, only after the enzyme is exposed to them in the presence of a high oxygen concentration for 10 to $20 \mathrm{~min}$. In the first type of incubation, we were studying the effect of acute exposure of the islets to the MAO inhibitors. During the early phases of the $15 \mathrm{~min}$ exposure to glucose (16.5 $\mathrm{mM}$ ), MAO would not be maximally inhibited by the irreversible MAO inhibitors. We hoped to detect alterations in insulin secretion that may not be related to the MAO inhibitory properties of these compounds. In the second type of incubation, the MAO inhibitors were in contact with the tissues in a $95 \%$ oxygen environment for $45 \mathrm{~min}$ prior to stimulation with $16.5 \mathrm{mM}$ glucose. In these studies MAO should have been maximally inhibited. With this design endogenous monoamines might accumulate in the islets of Langerhans, during the preincubation and incubation period.

When isolated pancreatic islets from obesehyperglycemic mice are incubated with 5-hydroxytryptophan this amino acid is taken up by the islets and converted to serotonin [20]. Under such experimental conditions, it is likely that L-dopa would be taken up by pancreatic islets and converted to dopamine [21]. Observations on the effect of pharmacological agents on insulin secretion support this concept [3]. There is more controversy concerning the uptake of monoamines by pancreatic islets. Following intraperitoneal injection into mice, histochemical tenchniques suggest that 5-HTP and L-dopa are more readily taken up by mouse B-cells than are serotonin and dopamine $[22,23]$. In contrast, isolated islets of obese-hyperglycemic mice take up serotonin as effectively as they take up 5-HTP [20, 24]. 5-HTP is a better inhibitor of insulin secretion from in vitro seg- ments of rabbit pancreas than is serotonin $[12,25]$. These diverse findings may be due to differences in species and experimental design. In the present studies we are assuming that 5-HTP enters the B-cells of the rabbit more effectively than does serotonin. MAO is predominantly located in the mitochondria of the $\mathrm{B}$-cell and the monoamines must be within the B-cell if they are to be metabolized by MAO. MAO inhibitors increase the inhibition of insulin secretion that occurs when pancreas segments are incubated with L-dopa, but they do not increase the inhibition of insulin secretion that occurs when the pancreas segments are incubated with dopamine [3]. The $45 \mathrm{~min}$ preincubation period that we employ also allows adequate time for the B-cell monoamine content to increase, particularly if MAO is inhibited.

We wished to use submaximal inhibition of insulin secretion by L-dopa and 5-HTP, so that we could detect increased inhibition of insulin secretion from MAO inhibition. In preliminary studies we determined that $0.01 \mathrm{mM} \mathrm{L-dopa}$ or $1 \mathrm{mM} 5$-HTP significantly inhibit insulin secretion in two thirds of the incubation experiments. A higher concentration of 5-HTP than of L-dopa may be necessary either because Dopa decarboxylase has a greater affinity for L-dopa than it does for 5-HTP [21] or because dopamine is a more potent inhibitor of insulin secretion than is serotonin [26].

\section{Results}

\section{$M A O$ Inhibitors and Insulin Secretion}

Fig. 1 depicts the results of 15 experiments that were designed to determine the effect of a series of MAO inhibitors on glucose-stimulated insulin secretion. The results are expressed as the percent of insulin secretion secreted by the control pieces, i.e., the pieces stimulated by $16.5 \mathrm{mM}$ glucose. Thus, if the MAO inhibitor does not alter glucose-stimulated insulin secretion, the insulin secretion will be $100 \%$ of control. In the left hand panel the MAO inhibitor was present only during stimulation with $16.5 \mathrm{mM}$ glucose. In the right hand panel the tissues were exposed to the MAO inhibitor for 45 min prior to stimulation with $16.5 \mathrm{mM}$ glucose, as well as during the $15 \mathrm{~min}$ stimulation period.

When the MAO inhibitor is present only during stimulation with glucose the following effects are noted: 1 and $0.1 \mathrm{mMHARM}$ potentiate insulin secretion; $0.1 \& 0.01 \mathrm{mM} \alpha$-MT potentiate insulin secretion; 1 mM TRAN inhibits insulin secretion; PARG does not alter insulin secretion; PHEN (1 mM), NIAL (1 mM) and IPRO $(0.1 \mathrm{mM})$ potentiate insulin secretion. 
When the MAO inhibitor is present during the 45 min preincubation period, as well as the 15 min incubation period with $16.5 \mathrm{mM}$ glucose, the following alterations are noted: HARM potentiates insulin secretion in a concentration of $0.1 \mathrm{mM}$ but not $1 \mathrm{mM}$; $\alpha$-MT $(1 \mathrm{mM})$ and TRAN $(1 \mathrm{mM} \& 0.1 \mathrm{mM})$ both inhibit insulin secretion; PARG does not alter insulin secretion; PHEN ( $1 \mathrm{mM})$ but not NIAL potentiate insulin secretion; and IPRO (1, 0.1 and $0.01 \mathrm{mM})$ potentiates insulin secretion. Despite the fact that it is not an MAO inhibitor, hydrazine (HYDRA) (1 and $0.01 \mathrm{mM}$ ) also potentiates insulin secretion.
Although the main purpose of the studies was to determine if MAO inhibitors alter glucose-stimulated insulin secretion we were also able to determine if exposure to the MAO inhibitor in the absence of a glucose stimulus altered insulin secretion. Forty-five minute exposure to $\alpha$-MT, TRAN, PARG, PHEN, NIAL, IPRO \& HYDRA (concentrations $1,0.1 \&$ $0.01 \mathrm{mM}$ ) did not alter insulin secretion from the pancreas segments, when the incubation medium contained $3.3 \mathrm{mM}$ glucose. HARM $(1 \mathrm{mM})$ increased insulin secretion above the basal level; basal release $0.7 \pm 0.20 \mu \mathrm{U} / \mathrm{mg} / 15 \mathrm{~min} ; \mathrm{HARM}(1 \mathrm{mM}) 4.1 \pm$

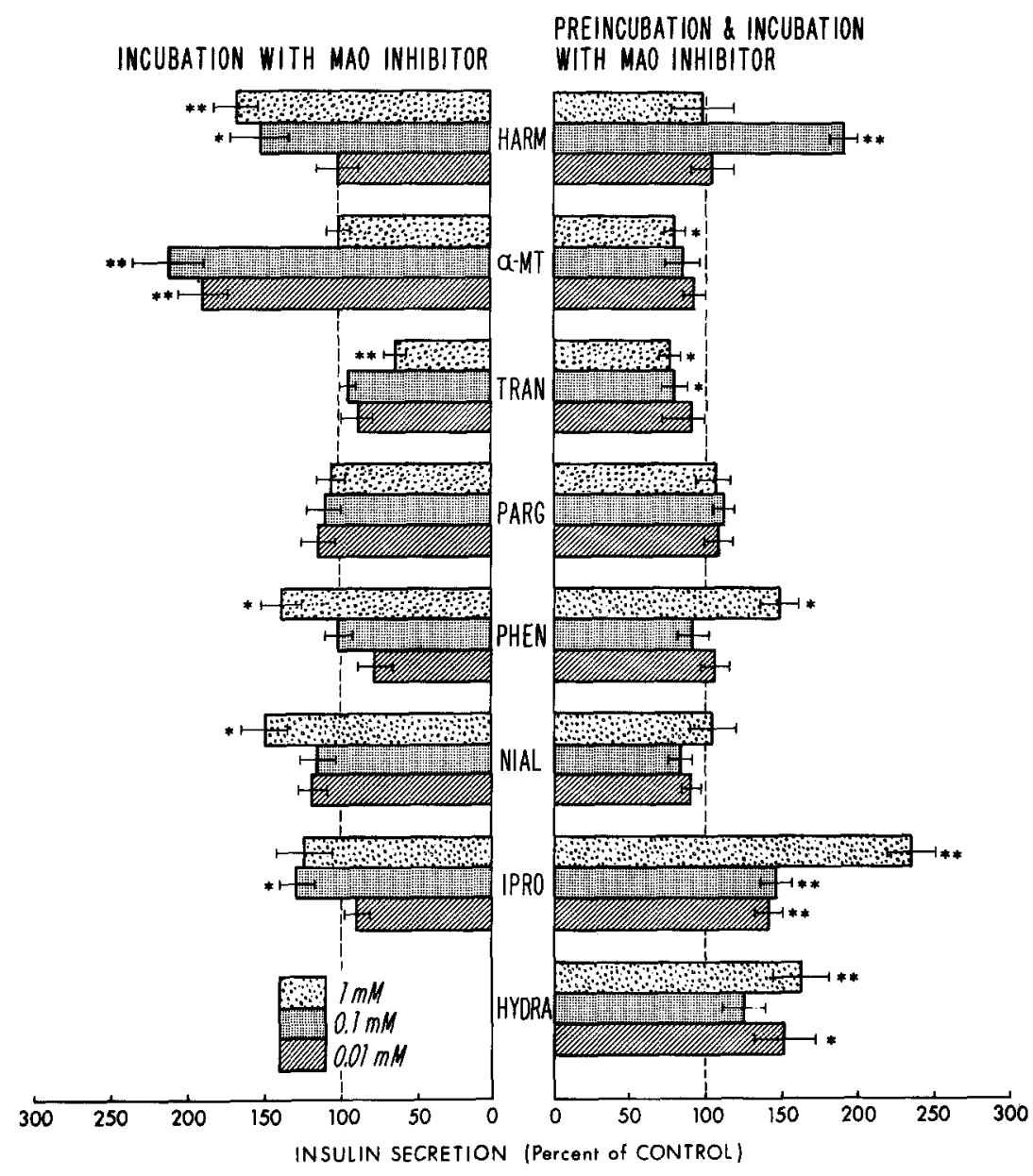

Fig. 1. Effect of MAO inhibitors on glucose-stimulated insulin secretion. In the incubation studies in the left hand panel, the MAO inhibitor was present only during the final 15 minute incubation period. In the incubation studies in the right hand panel, the MAO inhibitor was present both during the $45 \mathrm{~min}$ preincubation period and during the $15 \mathrm{~min}$ incubation period. Insulin secretion was stimulated with 16.5 $\mathrm{mM}$ glucose. Each bar represents the mean and the brackets \pm 1 SEM of 7 pancreas segments. The insulin secretion of each group of pancreas segments in an experiment is expressed as a percent of the mean insulin secretion of a group of 7 control pancreas segments incubated in $16.5 \mathrm{mM}$ glucose alone. The statistical significance of differences between experimental and control group are: ${ }^{* *} p<0.01$; and $* p<0.05$. The Mean \pm SEM absolute insulin secretion from the control segments in each experiment $(\mu \mathrm{U}$ insulin $/ \mathrm{mg} \mathrm{pancreas} / 15 \mathrm{~min})$ is incubation with MAO inhibitor - HARM $22.0 \pm 2.46, \alpha$-MT $14.2 \pm 1.02$, TRAN $9.7 \pm 0.34$, PARG $6.4 \pm 0.81$, PHEN $11.6 \pm 1.21$, NIAL $13.5 \pm 0.94$, IPRO $19.9 \pm 3.43$; preincubation and incubation with MAO inhibitors - HARM $26.4 \pm 2.16, \alpha-\mathrm{MT} 21.3 \pm 1.67$, TRAN $13.5 \pm 0.72$, PARG $22.3 \pm 2.79$, PHEN $6.1 \pm 0.69$, NIAL $27.3 \pm 3.98$, IPRO $24.7 \pm 3.09$, HYDRA $9.3 \pm 1.61$ 


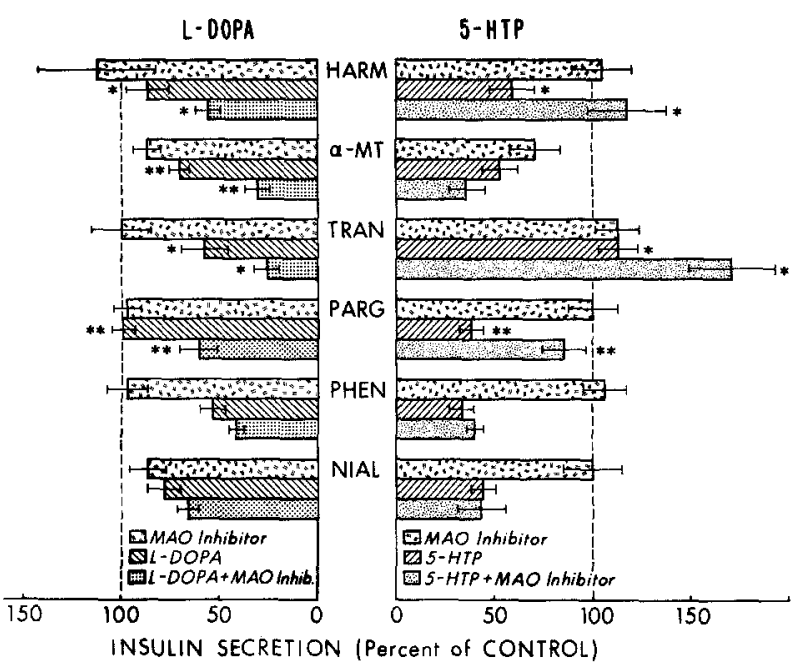

Fig. 2. Effect of MAO inhibitors on inhibition of insulin secretion by dopamine and serotonin. In the incubation studies in the left hand panel L-dopa $(0.01 \mathrm{mM})$ and the MAO inhibitor $(0.01 \mathrm{mM})$ were present during the $45 \mathrm{~min}$ preincubation period and the $15 \mathrm{~min}$ incubation period. In the incubation studies in the right hand panel 5-HTP $(1 \mathrm{mM})$ and the MAO inhibitor $(0.01 \mathrm{mM})$ were present during the $45 \mathrm{~min}$ preincubation period and the $15 \mathrm{~min}$ incubation period. Insulin secretion was stimulated by $16.5 \mathrm{mM}$ glucose. Each bar represents the mean and the brackets \pm 1 SEM of 7 pancreas segments. The insulin secretion of each group of pancreas segments in an experiment is expressed as a percent of the mean insulin secretion of a group of 7 control pancreas segments incubated in $16.5 \mathrm{mM}$ glucose alone. ** indicates two groups that differ from each other with $\mathrm{p}<0.01$. ${ }^{*}$ indicates two groups that differ from each other with $p<0.05$. The Mean \pm SEM absolute insulin secretion from the control group in each experiment ( $\mu \mathrm{U}$ insulin/ $\mathrm{mg} /$ pancreas/15 minutes) is: $L$-dopa - HARM 12.6 $\pm 2.48, \alpha$-MT $11.3 \pm 1.83$, TRAN $6.5 \pm 1.21$, PARG $18.7 \pm 1.40$, PHEN $12.8 \pm$ 1.97 , NIAL $17.4 \pm 1.36 ; 5-H T P-$ HARM $10.8 \pm 2.08, \alpha$-MT $14.6 \pm 2.61$, TRAN 14.6 \pm 1.66 , PARG $13.4 \pm 1.35$, PHEN 16.6 \pm 1.90, NIAL $16.1 \pm 3.08$

$0.75 \mu \mathrm{U} / \mathrm{mg} / 15 \mathrm{~min} ; \mathrm{HARM}(0.1 \mathrm{mM}) 1.1 \pm 0.25$ $\mu \mathrm{U} / \mathrm{mg} / 15 \mathrm{~min} ;$ HARM $0.01 \mathrm{mM}) 0.7 \pm 0.20$ $\mu \mathrm{U} / \mathrm{mg} / 15 \mathrm{~min}$. HARM $(1 \mathrm{mM})$ was a less potent stimulator of insulin release than glucose $16.5 \mathrm{mM}$, for in this experiment $16.5 \mathrm{mM}$ glucose stimulated $26.4 \pm$ $2.16 \mu \mathrm{U} / \mathrm{mg} / 15 \mathrm{~min}$.

Effect of MAO Inhibitors on Inhibition of Insulin Secretion by Dopamine and Serotonin. Fig. 2 depicts the results of a series of experiments to determine the effect of MAO inhibitors, monoamines and MAO inhibitors with monoamines on glucose stimulated insulin secretion. The design of all of these experiments was similar to those depicted in the right-hand panel of Figure 1, i.e. the MAO inhibitor and monoamine precursors were present in the 45 min preincubation period and in the $15 \mathrm{~min}$ incubation period. In the left-hand panel the pancreas segments were incubated with L-dopa (increasing B-cell dopamine) and in the right-hand panel they were incubated with 5-HTP (increasing the B-cell serotonin). In all of the experi- ments the concentration of MAO inhibitor used was $0.01 \mathrm{mM}$, for previous studies (Fig. 1, left panel) indicated that this concentration of MAO inhibitor usually did not itself alter insulin secretion when this experimental design was used. This was again confirmed, however, for each of the individual experiments in Fig. 2, for a $0.01 \mathrm{mM}$ concentration of the $\mathrm{MAO}$ inhibitor did not significantly alter insulın secretion stimulated by $16.5 \mathrm{mM}$ glucose in any of the experiments.

HARM, $\alpha$-MT, TRAN and PARG all significantly increased the inhibition of insulin secretion by L-dopa (B-cell dopamine). PHEN and NIAL caused a slight increase in dopamine inhibition of insulin secretion. but this change was not statistically significant. In contrast, none of the MAO inhibitors increased the inhibition of insulin secretion by 5-HTP (B-cell serotonin). HARM and PARG actually decreased inhibition of insulin secretion by serotonin. In the experiment performed with TRAN, 5-HTP alone did not inhibit insulin secretion. TRAN plus 5-HTP actually potentiated insulin secretion, i.e., the insulin secretion was greater than in the group incubated with TRAN and $16.5 \mathrm{mM}$ glucose (or the control group with $16.5 \mathrm{mM}$ glucose alone). $\alpha$-MT did not significantly alter serotonin inhibition of insulin secretion. Although the change was not significant, $\alpha$-MT itself caused a decrease in insulin secretion. The decrease in insulin secretion produced by serotonin and $\alpha$-MT may be an additive effect resulting from the inhibition by serotonin plus the inhibition by $\alpha$-MT. PHEN and NIAL did not alter serotonin inhibition of insulin secretion.

To try to determine if MAO inhibitors decrease serotonin inhibition of insulin secretion by decreasing 5-HTP uptake by the B-cells, we preincubated one group of pancreas segments in basal medium for 45 min and two other groups in medium containing $1 \mathrm{mM}$ 5-HTP. The B-cell serotonin was thus increased in the absence of an MAO inhibitor. There was no added 5-HTP in the final 15 minute incubation period, when the three groups were stimulated with $16.5 \mathrm{mM}$ glucose. PARG $(10 \mu \mathrm{M})$ was added to the medium of one of the groups that had been preincubated with 5-HTP. The glucose-stimulated insulin secretion of the control group was $7.2 \pm 1.16 \mu \mathrm{U} / \mathrm{mg} / 15 \mathrm{~min}$. The insulin secretion of the group preincubated in 5-HTP was significantly lower than the control group (3.8 \pm $0.37 \mu \mathrm{U} / \mathrm{mg} / 15 \mathrm{~min}, p<.05)$. The insulin secretion of the group preincubated with 5-HTP and then incubated with PARG was similar to the control group (6.9 $\pm 1.55 \mu \mathrm{U} / \mathrm{mg} / 15 \mathrm{~min}$ ). This study suggests that PARG exerts its effect at a point beyond the uptake of 5-HTP into the B-cell. 
Table 1. Specific activity of $M A O$ of rabbit islets, liver, kidney and pancreas using dopamine $(D A)$ and serotonin (5-HT) as substrates

\begin{tabular}{|c|c|c|c|c|c|}
\hline \multirow{3}{*}{$\begin{array}{l}\text { Substrate Conc. } \\
\text { Substrate }\end{array}$} & \multicolumn{5}{|c|}{ MAO Specific Activity $(\mu \mu \text { moles product } / \mathrm{mg} \text { protein } / \mathrm{min})^{\mathrm{a}}$} \\
\hline & \multicolumn{2}{|l|}{$28 \mu \mathrm{M}$} & \multicolumn{2}{|l|}{$14 \mu \mathrm{M}$} & $1.4 \mu \mathrm{M}$ \\
\hline & $\overline{\mathrm{DA}}$ & $5-\mathrm{HT}$ & $\overrightarrow{\mathrm{DA}}$ & $5-\mathrm{HT}$ & $5-\mathrm{HT}$ \\
\hline Islets & $357 \pm 62.3$ & $35 \pm 8.7$ & $104 \pm 14.1$ & $7 \pm 3.4$ & $7 \pm 2.0^{b}$ \\
\hline Liver & $808 \pm 53.9$ & $17 \pm 2.0$ & $462 \pm 65.4$ & $11 \pm 1.3$ & $49 \pm 12.7^{\circ}$ \\
\hline Kidney & $368 \pm 27.1$ & $43 \pm 6.8$ & $274 \pm 28.7$ & $24 \pm 5.5$ & $25 \pm 4.3$ \\
\hline Pancreas & $44 \pm 5.5$ & $22 \pm 3.5$ & $26 \pm 4.5$ & $15 \pm 2.0$ & $3 \pm 1.5^{b}$ \\
\hline
\end{tabular}

a Each number represents the Mean \pm SEM of tissues from 4 to 8 rabbits. The MAO specific activity against DA and 5-HT is significantly different $(p<0.02)$ with each tissue at each substrate concentration with the exception of pancreas at $14 \mu \mathrm{M}$.

b Not measurable.

Table 2. Effect of TRAN and HARM on MAO activity of rabbit liver

\begin{tabular}{|c|c|c|c|c|}
\hline \multirow{3}{*}{$\begin{array}{l}\text { Conc } \\
\text { TRAN } \\
\text { and } \\
\text { HARM } \\
\mu \mathrm{M})\end{array}$} & \multicolumn{4}{|c|}{ MAO Activity (Percent Control) } \\
\hline & $\overline{\mathrm{TRA}}$ & & HARM & \\
\hline & $\overline{\mathrm{DA}^{\mathrm{a}}}$ & $\overline{5-\mathrm{HT}^{\mathrm{a}}}$ & $\overline{\mathrm{DA}^{\mathrm{a}}}$ & $5-\mathrm{HT}^{\mathrm{a}}$ \\
\hline 100 & 1 & 3 & 4 & 5 \\
\hline 10 & 2 & 18 & 11 & 7 \\
\hline 1 & 7 & 26 & 50 & 11 \\
\hline 0.1 & 50 & 78 & 83 & 13 \\
\hline 0.01 & $b$ & $\mathrm{~b}$ & $\mathrm{~b}$ & 24 \\
\hline
\end{tabular}

a DA and 5-HT $14 \mu \mathrm{M}$

b Not Measured

SA against DA $394 \mu \mu$ moles $/ \mathrm{mg} / \mathrm{min}$

SA against 5 -HT $9 \mu \mu$ moles $/ \mathrm{mg} / \mathrm{min}$

\section{Substrate Specificity of Rabbit MAO}

Table 1 compares the specific activity of rabbit MAO for the substrates dopamine and serotonin. We studied the specific activity of islet, liver, kidney and pancreatic MAO at three substrate concentrations (28 $\mu \mathrm{M}, 14 \mu \mathrm{M}$, and $1.4 \mu \mathrm{M})$. The MAO activity of the tissue homogenates against serotonin was much less than the islet MAO activity against dopamine. The activities against serotonin $(28 \mu \mathrm{M})$ compared to the activities against dopamine $(28 \mu \mathrm{M})$ were: islets $10 \%$; liver $2 \%$; kidney $12 \%$; and pancreas $50 \%$. At $1.4 \mu \mathrm{M}$ serotonin, the activity of rabbit MAO activity against serotonin was below the level of sensitivity of the radioassay.

Additional studies were performed to determine if rabbit tissue MAO activity against both dopamine and serotonin is readily inhibited by MAO inhibitors. Homogenates of rabbit liver were incubated with dopamine $(14 \mu \mathrm{M})$ or serotonin $(14 \mu \mathrm{M})$ with varying concentrations of TRAN and HARM. Table 2, indi- cates that TRAN preferentially inhibits MAO activity against dopamine, while HARM preferentially inhibits MAO activity against serotonin. However, at $10 \mu \mathrm{M}$, the concentration used in the studies in Fig. 2, TRAN and HARM reduce the MAO activity against dopamine or serotonin to $18 \%$ of control or less.

\section{Discussion}

The present study indicates that different MAO inhibitors have diverse effects on glucose-stimulated insulin secretion from rabbit pancreas. Indeed, the same MAO inhibitor may have a different effect on insulin secretion when it is present only during glucose stimulation, from its effect when it is also present during the pre-stimulatory period (Fig. 1, $\alpha$-MT). Although potency may vary with the substrate that is used, the compounds investigated in general have the following order of decreasing potency as MAO inhibitors [27-30]: TRAN $>$ PHEN $>$ HARM $>$ PARG $>$ NIAL $>\alpha$-MT $>$ IPRO. Figure 1 indicates that there is a poor correlation between the potency of these compounds as MAO inhibitors and their ability to potentiate or inhibit glucose-stimulated insulin secretion.

HARM was the only non-hydrazine MAO inhibitor that potentiated glucose-stimulated insulin secretion when it was present during both the preincubation and incubation period. HARM also was the only MAO inhibitor that stimulated insulin secretion from pancreas segments incubated in medium with a low or non-stimulatory glucose concentration $(3.3 \mathrm{mM})$. In contrast, 3 of the 4 hydrazine-derivative MAO inhibitors we studied potentiated glucose-stimulated insulin secretion when they were present in the preincubation period and incubation period. NIAL was the only 
hydrazine-derivative MAO inhibitor that did not potentiate glucose-stimulated insulin secretion under these conditions. NIAL did potentiate insulin secretion when it was present only during the incubation period. This suggests that the ability of the hydrazinetype MAO inhibitors to potentiate insulin secretion may be related more to their hydrazine structure than to their MAO inhibitory properties. This suspicion was confirmed by incubating pancreas segments with hydrazine itself. Even at a concentration of $33 \mu \mathrm{M}$, hydrazine does not inhibit MAO activity [31]. In a concentration as low as $10 \mu \mathrm{M}$, hydrazine significantly potentiated insulin secretion (Fig. 1).

TRAN and $\alpha$-MT were the only MAO inhibitors that decreased insulin secretion when present during the preincubation and incubation period in the absence of added L-dopa. TRAN and $\alpha$-MT were also the only MAO inhibitors studied that are primary amines. Inhibition of insulin secretion by TRAN and $\alpha$-MT may be a direct effect of the compounds and may not be due to their MAO inhibitory properties, for other primary amines can inhibit insulin secretion $[32,33]$.

In addition to altering insulin secretion by mechanisms not related to MAO inhibition, MAO inhibitors may also modify insulin secretion by decreasing MAO activity. When B-cell dopamine was increased, by preincubating pancreas segments with L-dopa, HARM, $\alpha$-MT, TRAN and PARG increased the dopamine-inhibition of insulin secretion. We attribute this increased inhibition to an increase in the concentration of dopamine in the B-cells. In non-islet tissues, epinephrine and norepinephrine are primarily inactivated by catechol-O-methyl transferase (S-adenosylmethionine: catechol-O-methyl transferase, 2.1.6) [3] while dopamine is primarily inactivated by $\operatorname{MAO}[34$, 35]. MAO may be particularly important for inactivation of dopamine in the pancreatic islets of rabbits, for catechol-O-methyl transferase activity could not be demonstrated in this tissue [3, 4].

The failure of MAO inhibitors to potentiate the inhibitory effect of serotonin on insulin secretion was initially quite unexpected, for in most tissues MAO is the primary enzyme for inactivation of serotonin [36]. This might occur if MAO was not the primary route of serotonin inactivation in rabbit islets. We evaluated this possibility by determining the activity of rabbit MAO against dopamine and serotonin. The activity of rabbit MAO against dopamine was two (pancreas) to 50 (liver) times greater than against serotonin. Rabbit islets had 10 times more MAO activity against dopamine than serotonin. Indeed, we could not demonstrate MAO activity against serotonin when this monoamine was present in a $1.4 \mu \mathrm{M}$ concentration. Although the normal concentration of serotonin in rab- bit islets is not known, the serotonin concentration of rabbit liver and kidney is $3.6 \mu \mathrm{M}$ and $0.6 \mu \mathrm{M}$ respectively [37].

In addition to $\mathrm{MAO}$, serotonin can be inactivated by glucuronic acid conjugation or $\mathrm{N}$-acetylation [36]. In rabbit islets, the latter route may be more important than oxidative deamination by MAO. It is conceivable that MAO inhibitors do not potentiate serotonin-inhibition of insulin secretion because MAO does not play a major role in serotonin inactivation in rabbit islets.

In summary, the present study suggests that one way that MAO inhibitors can impair insulin secretion in the rabbit is by decreasing dopamine degradation in the pancreatic islets. In addition, however, MAO inhibitors also alter insulin secretion by mechanisms that do not involve MAO inhibition.

Acknowledgements. We thank Miss Connie Heiner for assistance in preparation of the manuscript.

\section{References}

1. Lebovitz, H. E., Feldman, J. M.: Pancreatic biogenic amines and insulin secretion in health and disease. Fed. Proc. 32, $1797-1802$ (1973)

2. Cegrell, L.: The occurrence of biogenic monoamines in the mammalian endocrine pancreas. Acta physiol. scand. (suppl.) 314, 1-26 (1968)

3. Feldman, J. M., Chapman, V.: Mechanisms of catecholamine inactivation by the endocrine pancreas. Diabetes $23,754-762$ (1974)

4. Feldman, J. M., Chapman, B.: Characterization of pancreatic islet monoamine oxidase. Metabolism 24, 581-588 (1975)

5. Bressler, R., Vargas-Cordon, M., Lebovitz, H. E.: Tranylcypromine: a potent insulin secretagogue and hypoglycemic agent. Diabetes 17, 617-624 (1968)

6. Gagliardino, J. J., Hernandez, R. E., Rodriguez, R. R., Lauri, H. C.: Stimulatory effect of nialamide on serum levels of insulin. Amer. J. Physiol. 219, 314-317 (1970)

7. Frohman, L. A.: Stimulation of insulin secretion in rats by pargyline and mebanazine. Diabetes 20, 266-270 (1971)

8. Potter, W. Z., Zaharko, D. S., Beck, L. V.: Possible role of hydrazine group in hypoglycemia associated with the use of certain monoamine-oxidase inhibitors (MAOI's). Diabetes 18, 537-541 (1969)

9. Lundquist, I., Ekholm, R., Ericson, I. E.: Monoamines in the pancreatic islets of the mouse, 5-hydroxytryptamine as an intracellular modifier of insulin secretion and the hypoglycemic action of the monoamine oxidase inhibitors. Diabetologia 7 , 414-422 (1971)

10. Alleyassine, H., Lee, S. H.: Inhibition by hydrazine, phenelzine and pargyline of insulin release from rat pancreas. Endocrinology 89, 125-129 (1971)

11. Alleyassine, H., Lee, S. H.: Inhibition of insulin release by substrates and inhibitors of monoamine oxidase. Amer. J. Physiol. 222, 565-569 (1972)

12. Feldman, J. M., Quickel, K. E., Lebovitz, H. E.: Potentiation of insulin secretion in vitro by serotonin antagonists. Diabetes 21 , 779-788 (1972) 
13. Feldman, J. M., Quickel, K. E.: Effects of amino acids, epinephrine and heparin upon the radioimmunoassay of insulin in plasma. Diabetes 22, 9-15 (1972)

14. Lacy, P. E., Kostianovsky, M.: Method for the isolation of intact islets of Langerhans from the rat pancreas. Diabetes 16, 35-39 (1967)

15. Hanks, J. H., Wallace, R. E.: Relation of oxygen and temperature in preservation of tissues by refrigeration. Proc. Soc. exp. Biol. (N. Y.) 71, 196-200 (1949)

16. Lowry, O. H., Rosenbrough, N. J., Farr, A. L., Randall, R. J.: Protein measurement with the Folin Phenol reagent. J. biol. Chem. 193, 265-275 (1951)

17. McCaman, R. E., McCaman, M. W., Hunt, J. M., Smith, M. S.: Micro determination of monoamine oxidase and 5-hydroxytryptophan decarboxylase activities in nervous tissues. J. Neurochem. 12, 15-23 (1965)

18. Zar, J. H.: Biostatistical Analysis. N. J.: Prentice-Hall Englewood Cliffs 1974

19. Zirkle, C. L., Kaiser, C.: Monoamine oxidase inhibitors (nonhydrazines) In: Psychopharmicological Agents, vol. I. (ed. M. Gordon) pp. 445-554. New York: Academic Press 1964

20. Gylfe, E., Hellman, B., Sehlin, J., Taljedal, I. B.: Amino acid conversion into 5-hydroxytryptamine in pancreatic B-cells. Endocrinology 93, 932 (1973)

21. Hagen, P.: Observations on the substrate specificity of dopa decarboxylase from adrenal medulla, human pheochromocytoma and human argentaffinoma. Brit. J. Pharmacol. 18, 175-182 (1962)

22. Cegrell, L., Falck, B., Hellman, B.: Monoaminergic mechanisms in the endocrine pancreas. In: The structure and metabolism of pancreatic islets (eds. S. E. Brolin, B. Hellman, H. Knutson) pp. 429-435. Oxford: Pergamon Press 1964

23. Cegrell, L.: Monoaminergic mechanisms in the pancreatic $\alpha$-cells. In: The structure and metabolism of the pancreatic islets (eds. S. Falkner, B. Hellman, I. B. Taljedal) pp. 131-140. Oxford: Pergamon Press 1970

24. Hellman, B., Lernmark, A., Sehlin, J., Taljedal, I. B.: Transport and storage of 5-hydroxytryptamine in pancreatic B-cells. Biochem. Pharmacol. 21, 695-706 (1972)

25. Feldman, J. M., Lebovitz, H. E.: A serotoninergic mechanism for the control of insulin secretion. Trans. Ass. Amer. Physcns. 85, 279-294 (1972)

26. Quickel, K. E., Feldman, J. M., Lebovitz, H. E.: Inhibition of insulin secretion by serotonin and dopamine: species variation. Endocrinology 89, 1295-1302 (1971)

27. Udenfriend, S., Witkop, B., Redfield, B. G., Weissbach, H.: Studies with reversible inhibitors of monoamine oxidase: harmaline and related compounds. Biochem. Pharmacol. 1, 160-165 (1958)

28. Greig, M. E., Walk, R. A., Gibbons, A. J.: The effect of three tryptamine derivatives on serotonin metabolism in vitro and in vivo. J. Pharmacol. exp. Ther. 127, 110-115 (1959)

29. Maxwell, D. R., Gray, W. R., Taylor, E. M.: Relative activity of some inhibitors of monoamine oxidase in potentiating the action of tryptamine in vitro and in vivo. Brit. J. Pharmacol. 17, 310-320 (1961)

30. Hendley, E., Snyder, S. S.: Relationship between the action of monoamine oxidase inhibitors on the noradrenaline uptake system and their antidepressant efficacy. Nature 220, 1330-1331 (1968)

31. Gomes, B., Igaue, I., Kloepfer, H. G., Yasunobu, K. T.: Amine oxidase XIV. Isolation and characterization of the multiple beef liver amine oxidase components. Arch. Biochem. 132, 16-27 (1969)

32. Feldman, J. M., Lebovitz, H. E.: Structural determinants of catecholamine action on in vitro insulin release. J. Pharmacol. exp. Ther. 176, 611-621 (1971)

33. Feldman, J. M., Lebovitz, H. E.: Structural determinants of indole amine action on in vitro insulin release. Endocrinology 91, 809-816 (1972)

34. Blaschko, H.: Amine oxidase and amine metabolism. Pharmacol. Rev. 4, 41.5-458 (1952)

35. Gatgounis, J.: Structural activity relationships of a series of amines injected before and after monoamine oxidase inhibitor. Arch. int. Pharmacodyn. 154, 412-420 (1965)

36. McIsaac, W. M., Page, I. H.: The metabolism of serotonin (5-hydroxytryptamine). J. Biol. Chem. 234, 858-864 (1959)

37. Kakimoto, Y., Armstrong, M. D.: On the identification of octopamine in mammals. J. Biol. Chem. 237, 422-427 (1962)
J. M. Feldman, M. D.
Division of Endocrinology
Duke Univ. Medical Center
Box 2963
Durham, North Carolina 27710
USA 